\title{
Ion plasma waves induced by frustrated Debye shielding
}

\author{
R. P. Drake \\ University of Michigan, Ann Arbor, Michigan 48109 \\ R. S. Marjoribanks \\ University of Toronto, Toronto, Ontario M5S 1A7, Canada
}

(Received 12 September 2001; accepted 15 October 2001)

\begin{abstract}
The oscillation of electrons, in a sufficiently intense pump wave, frustrates Debye shielding in the direction of the oscillation. One finds that such oscillating electrons cannot shield charge fluctuations over distances smaller than the distance they sample in a plasma period. One consequence is that the frequency of ion waves can be increased from the ion acoustic frequency to the ion plasma frequency in the presence of large enough oscillations. This may explain a number of observations in laser experiments. More generally, any phenomenon involving Debye shielding will be altered by an intense pump wave. (C) 2002 American Institute of Physics.
\end{abstract}

[DOI: $10.1063 / 1.1425839]$

\section{INTRODUCTION}

Debye shielding, introduced in the first lecture of the typical course on plasma physics, is one of the most fundamental properties of plasmas. The charges in the plasma move so as to shield the electric field produced by any single charge or by any charged object. The corresponding structure of the electric field has many effects. For example, Debye shielding affects the frequency of ion waves, ${ }^{1}$ the rates of atomic transitions, ${ }^{2}$ bremsstrahlung interactions, ${ }^{3}$ synchrotron radiation, ${ }^{4}$ and Stark broadening. ${ }^{5}$ This implies that any process that alters the Debye shielding behavior of plasmas may have many and far-reaching consequences. We identify and discuss one such process here: the frustration of Debye shielding by an intense electromagnetic wave.

We explore this issue by examining the behavior of longitudinal ion fluctuations in plasmas. These are present in all media containing ions, but in plasmas they are not necessarily acoustic waves. Acoustic waves occur, in plasmas, when the electrons can screen the fluctuations of the ion charge density by Debye shielding, so that the pressure gradient drives the oscillation. When Debye shielding becomes ineffective, electrostatic restoring forces dominate, and iondensity fluctuations oscillate at the ion-plasma frequency instead of the much smaller acoustic frequency. The waves are then ion-plasma waves, long understood ${ }^{1,6}$ and recently observed $^{7,8}$ in thermal, unmagnetized plasmas. In this paper we show that Debye shielding can be frustrated by large, high-frequency electron oscillations, and that this can induce waves that would be ion-acoustic waves to become ionplasma waves. These waves are more weakly damped than the acoustic waves. They can be unstable. Their frequency and growth rate can be large enough that they can be driven to large amplitude in $100 \mathrm{fs}$ laser-plasma experiments, unlike ordinary acoustic waves. This may explain the observation of ion-plasma-frequency satellites in such experiments, as well as other phenomena in both ps and ns experiments.

In the following, we first provide a physical discussion of ion-plasma waves and of how an intense pump can in- crease the frequency of ion-acoustic waves to frequencies near the ion-plasma frequency, producing waves that we designate as induced ion-plasma waves. Then we review the relevant literature, after which we derive a fluid theory describing the wave behavior. We then apply this theory to some cases, and compare its results to those of published kinetic-theory results in one limiting case, after which we conclude.

\section{PHYSICAL DISCUSSION}

Consider ion waves in an unmagnetized, isolated, equilibrium plasma. The ion density fluctuates at some frequency, $\omega$, and with some wave number, $k$. A continuous dispersion relation ${ }^{1}$ connects the ion-wave behavior between the acoustic regime and the ion-plasma-wave regime. The waves are acoustic waves when $k \lambda_{D} \ll 1$. (Here $\lambda_{D}$ is the electrostatic shielding distance of the electrons, the electron Debye length.) The waves become ion-plasma waves, with $\omega$ near the ion-plasma frequency, $\omega_{p i}$, when $k \lambda_{D} \sim 1$. Having $k \lambda_{D}$ $\sim 1$ has the consequence the electrons are unable to cluster themselves more finely than the scale of the ion-densityfluctuation wavelength. As $k$ increases from the acoustic regime toward the ion-plasma-wave regime, the phase velocity of the ion waves decreases below the acoustic sound speed, since their frequency is clamped near $\omega_{p i}$. In consequence, such ion-plasma waves are strongly damped unless the ion thermal velocity is far below the acoustic sound speed, which occurs when $Z T_{e} / T_{i}$ is quite large, ${ }^{8}$ where $Z$ is the average ion charge and $T_{e}$ and $T_{i}$ are the electron and ion temperatures, respectively. Ion plasma waves resemble, in some respects, ion Langmuir waves in non-neutral, pure-ion plasmas $^{9}$ and lower-hybrid waves propagating perpendicular to an ambient magnetic field. In both these cases, the fluctuations of the ion charge density are also unshielded.

This discussion prepares us to understand the impact, on ion waves, of large electron oscillations. In the oscillating electric field of a pump, the electrons oscillate with velocity $\mathbf{v}_{\text {osc }}=\mathbf{v}_{\text {os }} \cos \left(\mathbf{k}_{0} \cdot \mathbf{x}-\omega_{0} t\right)$. Here we take the pump wave, of 
wave vector $\mathbf{k}_{0}$ and frequency $\omega_{0}$, to have an electric field that oscillates in the $z$ direction. The amplitude of the oscillating velocity is related to the amplitude of the electric field of the light wave, $E_{0}$, by $v_{\text {os }}=e E_{0} / m \omega_{0}$, so long as $v_{\text {os }}$ $\ll c$, where $e$ and $m$ are the charge and mass, respectively, of the electron and $c$ is the speed of light. The ion oscillations are negligible by comparison and will be ignored. The amplitude of the spatial excursion of the electrons is $x_{\mathrm{os}}$ $=v_{\mathrm{os}} / \omega_{0}$.

Here is the crucial physical point: an intense pump frustrates the ability of the electrons to shield ion density modulations along the direction of the pump field. Heuristically, electrons adapt to electrostatic perturbations at a maximum rate $\omega_{\text {pe }}$ their characteristic cutoff frequency. Electrons dragged by the laser at speed $v_{\text {os }}$ through the ion-wave background see electrostatic fluctuations at frequency $k v_{\text {os }}$. In consequence, ion waves propagating in the direction of the electron oscillations must become ion plasma waves as $k v_{\text {os }} / \omega_{\text {pe }}$ approaches 1 .

\section{PRIOR WORK}

Past studies of strong pump effects on waves have included both fluid theory ${ }^{10-12}$ and kinetic theory. ${ }^{10,11,13-17}$ The important point about such prior work is that none of it has identified the fact that $\omega_{p i}$ is a limiting frequency for driven waves, or has discussed the physical connection to ionplasma waves. These points are essential when considering the behavior of plasmas driven by the intense, short-pulse lasers of today. In addition, all the prior calculations produce actual results only under the constraint $k \gg k_{0}$. The resulting models can be described as "dipole pump" or "nonpropagating pump" models. Present-day experiments violate this constraint.

First consider the kinetic-theory papers that treat the strong-pump case. ${ }^{10,11,13-17}$ The requirement that $k \gg k_{0}$ is essential to the derivations in these papers, which cannot be extended to the general case. ${ }^{18}$ However, we very much need to escape the limitation $k \gg k_{0}$, because present-day experiments violate it. In addition, there are two philosophical reasons why it seems that one might be able to escape it. First, the fundamental, qualitative dynamics described above does not depend upon the spatial wavelength of the driving electric field, so it should be possible to develop a model that escapes this constraint. Second, ion waves are slow phenomena that average the electron behavior over very many electron-plasma periods. One should not require kinetic theory for the electrons in order to capture the essential dynamics of such a process. This is the justification for hybrid simulation codes, for example, which treat particle ions and fluid electrons. Electron kinetic theory may prove necessary, however, to obtain exact results when ion waves are unstably coupled to strongly damped Langmuir waves, as in some past experiments. ${ }^{19,20}$

There have been many fluid treatments of ion waves, but only a few have considered the strong-pump case. ${ }^{10-12}$ Some of these, such as that of Kaw and Dawson, ${ }^{12}$ begin with completely general fluid equations. In principle such studies could have been extended to propagating pumps, although it appears that some of them might have missed the parametric coupling to Langmuir waves, discussed below. In addition, any fundamentally correct treatment of the electron susceptibility must include the effect of oscillations. Any calculation that obtains unstable mode coupling without finding some modification to the electronic susceptibility cannot be correct in the limit of large oscillations. We refer here to the physical susceptibility, not to the standard integral (which is this susceptibility in the limit of very simple plasmas). Other fluid theory treatments, such as those cited here, ${ }^{21,22}$ begin with an acoustic wave equation. This is correct only in the limit of small pump and of $k \lambda_{D} \ll 1$, which is not the case of interest here. Here we develop a fluid theory that naturally allows for a large-amplitude, propagating pump, as is present in current experiments.

\section{FLUID THEORY}

Our approach is to derive the electron fluid equations in the presence of large electron oscillations, but with the aim of understanding the low-frequency response of the plasma. Our goal, in this nonrelativistic, homogeneous-plasma model, is to determine the essential response of the local ion waves to a strong pump. We expect that this will help one understand the qualitative features present in more complicated systems, such as those with steep gradients or relativistic oscillation velocities.

We begin with the nonrelativistic Vlasov equation for the electron distribution function, $f$,

$$
\frac{\partial f}{\partial t}+\mathbf{v} \cdot \frac{\partial f}{\partial \mathbf{x}}+\frac{-e}{m}\left|\mathbf{E}_{t}+\frac{\mathbf{v}}{c} \times \mathbf{B}\right| \cdot \frac{\partial f}{\partial \mathbf{v}}=0,
$$

and we take the first two moments of this equation in velocity to obtain continuity and momentum equations. Here $f$ is the distribution function in the presence of the total electric field, $\mathbf{E}_{t}$, which includes the high-frequency components. The key to obtaining useful moments, in the presence of the high-frequency waves, is to recognize the symmetry that may be present in $f$. In the absence of any waves or any average drift velocity, $f(\mathbf{v})$ may be assumed to be symmetric about $\mathbf{v}=0$. In the event that there is an average drift velocity, $\mathbf{u}$, then $f$ is symmetric in the variable $(\mathbf{v}-\mathbf{u})$. Now any oscillating electric fields that may be present cause all the electrons to oscillate equally. (This is the reason why it can be effective to undertake a kinetic theory in an oscillating frame, but as discussed above this only turns out to be useful in the limit that $k \gg k_{0}$.) Here we can recognize that, in the presence of high-frequency electric fields, the electron distribution function $f$ is symmetric in the variable $\mathbf{g}=(\mathbf{v}-\mathbf{u}-\mathbf{w})$, where $\mathbf{u}$ is the average drift velocity of the electron fluid and $\mathbf{w}=\mathbf{v}_{\text {osc }}+\mathbf{w}_{\mathbf{1}}$ is the net oscillating velocity due to all the high-frequency waves present. Here $\mathbf{w}_{\mathbf{1}}$ includes all such modes other than the pump, and is taken to be of small amplitude compared to $v_{\text {os }}$. In making the assumption that $f$ is symmetric in $\mathbf{g}$, we are assuming that any asymmetries that do arise in the electron distribution function will be unimportant for ion waves. In nearly all circumstances, this is a good assumption. The moments of $f$, which matter for ion waves, are 


$$
\int f(\mathbf{g}) d \mathbf{g}=n, \int g_{i} f(\mathbf{g}) d \mathbf{g}=0,
$$

and

$$
\int g_{i}^{2} f(\mathbf{g}) d \mathbf{g}=n T_{e} / m,
$$

where $n$ is the electron density, $g_{i}$ is any component of $\mathbf{g}$, and we take the second moment of $f$ to define the temperature (in energy units) as shown. ${ }^{23}$

After taking these moments, we average over many oscillations of the high-frequency electric field. Designating averages by \langle\rangle , we obtain $\langle\mathbf{w}\rangle=0$ and $\left\langle\mathbf{v}_{\text {osc }}^{2}\right\rangle=v_{\text {os }}^{2} / 2$. The averaging of the electric field leaves only any low-frequency component from the ion waves, which we henceforth label E. We are left with a continuity equation and with a momentum equation. As usual, the continuity equation is used to simplify the momentum equation. We note that this is most conveniently done after the time averaging, to avoid producing terms whose averaging is not straightforward. One thus obtains

$$
m n \frac{\partial \mathbf{u}}{\partial t}+m n \mathbf{u} \cdot \nabla \mathbf{u}=-e n \mathbf{E}-\nabla \cdot \mathbf{P}-\frac{e n}{c}\langle(\mathbf{u}+\mathbf{w}) \times \mathbf{B}\rangle,
$$

in which the pressure tensor is given by

$$
\mathbf{P}=n T_{e} \mathbf{I}+m\langle n \mathbf{w} \mathbf{w}\rangle .
$$

We note that to this point we have assumed symmetry in $f$ as described above, and have assumed the validity of the nonrelativistic Vlasov equation, but have made no smallness assumptions.

The reader may recognize that both the term, $m\langle n \mathbf{w w}\rangle$, in Eq. (4) and some portion of the final term in Eq. (3) contribute to the "ponderomotive force," as usually defined. For this application, it is essential to correctly treat the derivative of density present in $\nabla \cdot \mathbf{P}$. It is this derivative that expresses the loss of response of the electron fluid to fluctuations along the direction of $\mathbf{v}_{\mathrm{os}}$, even if $v_{\mathrm{os}}^{2}$ is constant in space. This is the largest factor through which such oscillations affect the ion-wave frequency. This factor will also affect the average field structure produced by Debye shielding in general, with potential consequences for the other applications mentioned in the Introduction.

At this point, an aside is essential for some readers, who will recall that the traditional results of kinetic theory involve Bessel functions, and who may be expecting them to arise here. The Bessel functions in the traditional theory arise from a calculation involving two steps described very clearly in San Martin. ${ }^{15}$ The first step is to assume that the pump is a dipole pump and then to transform the position and velocity variables so as to obtain the equations that apply in a frame of reference that oscillates with the electrons. If the vector corresponding to the electron oscillations in space is $\boldsymbol{\epsilon}$ $=\mathbf{v}_{\text {os }} / \omega_{0}$, then this substitution involves taking a new position variable, equal to $\mathbf{x}+\boldsymbol{\epsilon} \sin \left(\omega_{0} t\right)$, and a new velocity variable, equal to $\mathbf{v}+\omega_{0} \boldsymbol{\epsilon} \cos \left(\omega_{0} t\right)$. The second step is to Fourier transform the resulting equations in space. This generates factors of the form $\exp \left[-i \mathbf{k} \cdot \boldsymbol{\epsilon} \sin \left(\omega_{0} t\right)\right.$,], for which the following identity introduces the Bessel functions,

$$
e^{-i \mathbf{k} \cdot \boldsymbol{\epsilon} \sin \left(\omega_{0} t\right)}=\sum_{p} J_{p}(\mathbf{k} \cdot \boldsymbol{\epsilon}) e^{i p \omega_{0} t} .
$$

The important point here is that there is nothing whatsoever unique to kinetic theory about this aspect of the traditional calculation. If one begins with the fluid equations, assumes a dipole pump, and undertakes the same two steps, then one arrives at the same series of Bessel functions. However, if one breaks the assumption of a dipole pump, and performs the same two steps, then one does not obtain a result involving Bessel functions. (This point is also discussed in the Ph.D. thesis of Phillipe Mounaix. ${ }^{18}$ ) In fact, the kinetic theory equations in this case become quite intractable, which further motivated the fluid approach undertaken here. In conclusion, the sum involving the Bessel functions is a general consequence of the assumption of a dipole pump, but does not in any sense represent a general property of kinetic theory. One can recover this sum by making the appropriate assumptions from a certain point forward in our derivation, but one will not obtain it as a limit of our final result. (Kaw and Dawson, who do assume a dipole pump in their fluid theory, do obtain a sum over Bessel functions. ${ }^{12}$ )

In exploring the specific effect on ion waves, one can proceed to derive linearized electron and ion wave equations. As usual, this involves the assumptions that the fluids can be treated as polytropic gases, and that the fluctuations of the density are small compared to the initial density. One obtains the intermediate step,

$$
\begin{aligned}
\frac{\partial^{2} \tilde{n}}{\partial t^{2}} & +\omega_{\mathrm{pe}}^{2} \tilde{n}-\left[\frac{T_{e}}{m} \nabla^{2}+\frac{v_{\mathrm{os}}^{2}}{2} \frac{\partial^{2}}{\partial z^{2}}\right] \tilde{n}-\omega_{\mathrm{pe}}^{2} \tilde{N} \\
& =-\nabla \cdot \nabla \cdot\langle\mathbf{w} \mathbf{w}\rangle-\nabla \cdot\langle\mathbf{w} \times \nabla \times \mathbf{w}\rangle,
\end{aligned}
$$

and

$$
\frac{\partial^{2} \widetilde{N}}{\partial t^{2}}+\omega_{p i}^{2} \tilde{N}-\frac{3 T_{i}}{M} \nabla^{2} \tilde{N}=\omega_{p i}^{2} \tilde{n} .
$$

Here $\omega_{\mathrm{pe}}$ is the electron plasma frequency, $M$ is the ion mass, and the ion-wave fluctuation amplitude of the electron or ion density, normalized to its average density, is $\tilde{n}$ or $\tilde{N}$, respectively. Only first order terms are kept on the right-hand side and only low-frequency terms survive the averaging. The right-hand side of Eq. (6) includes the ponderomotive force terms through which other (longitudinal or transverse) waves are coupled to the ion waves by the (longitudinal or transverse) pump. The left-hand side of Eq. (6) includes the pump-induced loss of response to fluctuations (the term proportional to $v_{\mathrm{os}}^{2}$ ). This term usually has not been present in prior work, and its importance has never been discussed. Nonetheless, this term has a dominant influence on the behavior of ion modulations in the presence of strong pump waves. As should be the case, Eq. (6) has a clear and logical connection to results that have been found previously. One can obtain its left-hand side by time averaging Eq. (11b) of Kaw and Dawson ${ }^{12}$ (and ignoring damping). Its right-hand side is consistent with past fluid treatments of parametric 
decay $^{24,25}$ (which used ion waves that were acoustic by assumption). Equation (7) has often been found previously, again for example in Kaw and Dawson.

One can then Fourier transform Eqs. (6) and (7) and solve them, recognizing that $\omega / \omega_{\text {pe }}$ can be neglected, where $\omega$ is the ion wave frequency. Unfortunately, whenever the term proportional to $v_{\mathrm{os}}^{2}$ on the left-hand side of Eq. (6) is important, the terms on the right-hand side also matter. These include terms that arise from three-wave coupling. Specifically, the ion wave beats with the pump to drive both downshifted and upshifted high-frequency Langmuir waves, known as Stokes and anti-Stokes modes, respectively. These in turn beat with the pump and affect the ion wave. Under some circumstances, the ion wave, the Stokes mode, and the anti-Stokes mode all grow simultaneously, extracting energy from the pump. A "4-wave" analysis ${ }^{24,26-29}$ can treat this case in addition to the simpler " 3 -wave" case ${ }^{25,30}$ that arises when the anti-Stokes mode is not important.

From the high-frequency electron fluid equations, one can find the amplitudes of the Langmuir modes driven by beating of the ion wave and the pump. The amplitudes of these Stokes and anti-Stokes modes are given by

$$
D_{S}\left(\mathbf{k}_{0}-\mathbf{k}_{S}, \omega_{0}-\omega^{*}\right) \tilde{n}_{S}=C_{S i} \tilde{n}^{*}
$$

and

$$
D_{A}^{*}\left(\mathbf{k}_{0}+\mathbf{k}_{A}, \omega_{0}+\omega\right) \tilde{n}_{A}^{*}=C_{A i} \tilde{n}^{*},
$$

respectively, where

$$
D_{S}\left(\mathbf{k}_{q}, \omega\right)=D_{A}\left(\mathbf{k}_{q}, \omega\right)=\omega^{2}-\omega_{\mathrm{pe}}^{2}-3 k_{q}^{2} v_{\mathrm{th}}^{2}
$$

and

$$
C_{S i}\left(\mathbf{k}_{q}\right)=-C_{A i}\left(\mathbf{k}_{q}\right)=-\left(\mathbf{k}_{q} \cdot \mathbf{v}_{\mathrm{os}}\right) / 2 .
$$

Here $\widetilde{n}_{S}$ and $\tilde{n}_{A}$ are the normalized, complex fluctuation amplitudes (in phase space) of the Stokes and anti-Stokes Langmuir waves, respectively; $k_{S}$ and $k_{A}$ are the wave number (or in boldface, wave vector) of the longitudinal Stokes and antiStokes modes, respectively, with corresponding subscript $S$ or $A$; and $q$ can designate either $S$ or $A$ as appropriate. [We work in the common complex notation ${ }^{31}$ in which a real quantity is represented by $\operatorname{Re}(H)=\left(H+H^{*}\right) / 2$.]

Solving Eqs. (6) and (7) for the electron-density fluctuations associated with the ion waves, one obtains the relation,

$$
D_{i}^{*}(\mathbf{k}, w) \tilde{n}^{*}=C_{i S} \tilde{n}_{S}+C_{i A} \tilde{n}_{A}^{*},
$$

in which the coupling coefficients are

$$
C_{i S}=\frac{-\omega_{p i}^{2}}{\omega_{\mathrm{pe}}^{2}} \frac{\left(\mathbf{k} \cdot \mathbf{v}_{\mathrm{os}}\right)\left(k^{2}+k_{S}^{2}\right)}{2\left(1+k^{2} \lambda_{D}^{2}+k_{z}^{2} \lambda_{S}^{2}\right)^{2}} \frac{\omega_{S}}{k_{S}^{2}}
$$

and

$$
C_{i A}=-C_{i S} \frac{\left(k^{2}+k_{A}^{2}\right)}{\left(k^{2}+k_{S}^{2}\right)} \frac{k_{S}^{2}}{k_{A}^{2}} \frac{\omega_{A}}{\omega_{S}} \approx-C_{i S},
$$

and in which the ion-wave dispersion function is

$$
D_{i}=\omega^{2}-3 k^{2} \frac{T_{i}}{M}-\frac{\omega_{p i}^{2}\left(k^{2} \lambda_{D}^{2}+k_{z}^{2} \lambda_{S}^{2}\right)}{\left(1+k^{2} \lambda_{D}^{2}+k_{z}^{2} \lambda_{S}^{2}\right)},
$$

where we have introduced a new shielding distance, $\lambda_{S}$ $=F_{s} v_{\mathrm{os}} / \omega_{\mathrm{pe}}$. This is the anticipated shielding due to the electron oscillations in the strong electric field. Here $k_{z}$ is the component of $\mathbf{k}$ in the $z$ direction, while $F_{s}$ is a factor of value near unity, given for a transverse pump by

$$
F_{s}=\sqrt{1+\frac{1}{2} \frac{k^{2}\left(k^{2}+k_{0}^{2}\right)}{\left(k^{2}+k_{0}^{2}\right)^{2}-4\left(\mathbf{k} \cdot \mathbf{k}_{0}\right)^{2}}} .
$$

Equation (12) is the key result in the present paper. It shows how low-frequency fluctuations behave in the presence of a strong pump. We apply this result to some cases of interest in the next section.

Before turning to applications, we discuss the limits of validity of the above theoretical results, and the anticipated impact of departures from strict validity. The first limitation involves relativistic effects. The above equations are nonrelativistic. They thus do not apply as written to any system in which the oscillating velocity in any wave (or in any combination of waves) approaches $c$. In addition, one must require that $T_{e} \ll m c^{2}$ and $T_{i} \ll M c^{2}$. In the relativistic limit, the qualitative conclusion reached here will still be correct-ion waves of sufficiently large $k$ will become ion plasma waves. One might guess, based on the above reasoning that the threshold for this transition would be approximately $k$ $>\omega_{\mathrm{pe}} / c$. However, the variation of the ion-wave wavelength, as seen in the rapidly changing rest frame of the electrons, might lead to a different result. We hypothesize that the threshold $k$ will be reduced, but leave the evaluation of this case to future work.

The second limitation involves kinetic effects. The above equations will become inaccurate when kinetic effects alter the frequencies of the modes involved or introduce damping large enough to affect the coupling of the modes. In these cases, the structure of Eqs. (8)-(15) does not change. What does change is that $D_{i}, D_{S}$, and $D_{A}$ as given may become inaccurate for the three waves in the plasma. If the coupling to the Langmuir waves is not important, then the ion wave becomes strongly damped when its phase velocity decreases to of order the ion thermal velocity. This may never occur if $Z T_{e} / T_{i}$ is large enough, as may be true for some cases of interest. If $Z T_{e} / T_{i}$ is not too large, then we will see that the ion wave becomes strongly damped at smaller $k \lambda_{D}$ in the presence of a strong pump than in the case without a pump. Kinetic theory for the case without a pump shows that the real part of the wave frequency in this case does not strongly differ from the fluid result. ${ }^{6}$ We see no reason to expect otherwise here. If the coupling to Langmuir waves is important, then $D_{S}$ and $D_{A}$ become inaccurate when $k \lambda_{D} \geqslant 0.3$. However, the difference in the real part of the frequency of these Langmuir waves is modest enough, and has a small enough effect on the solution of Eqs. (8)-(15) that we expect the theory above to give qualitatively correct results even in this limit.

\section{APPLICATIONS}

First we consider the behavior at densities far below the critical density, $n_{c}$, where the Langmuir-wave coupling is weak. In this case, the properties of the ion waves may be 


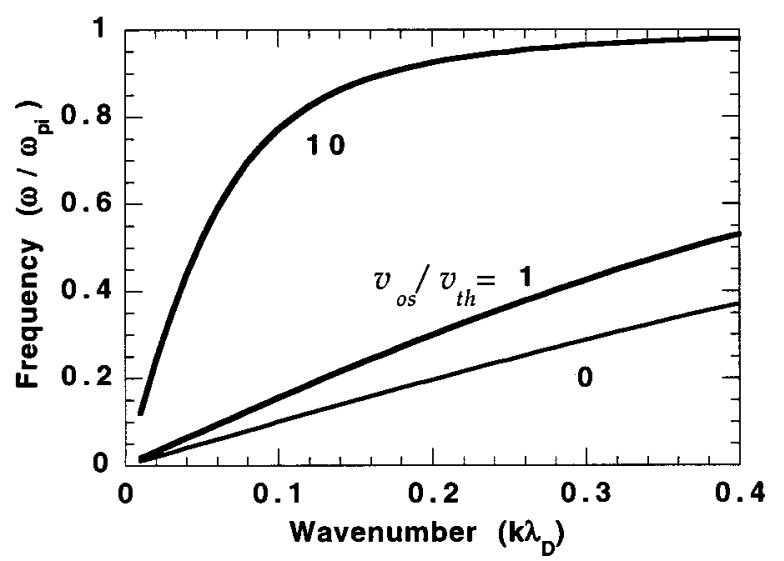

FIG. 1. The relation of frequency and wave number for the induced ion plasma wave dispersion relation $\left(D_{i}=0\right)$ is shown for the indicated values of $v_{\text {os }} / v_{\text {th }}$. As is discussed in the text, the ion temperature is assumed to be negligible here and in the other figures.

found by setting $D_{i}=0$. Such waves might be driven to large amplitude by stimulated scattering. Figure 1 shows the corresponding dependence of $\omega / \omega_{p i}$ on $k \lambda_{D}$ for three values of $v_{\text {os }} / v_{\text {th }}$ with $v_{\text {th }}=\sqrt{T_{e} / m}$. Figure 1 assumes $\mathbf{k} \| \mathbf{v}_{\text {os }}$ and $k$ $=3 k_{0}$ (neither of which strongly affect $\omega$ ), and also assumes $T_{i}$ is negligible, as should be the case in short-pulse laser experiments. One can see how the electron oscillations act to increase both the frequency and the phase velocity of the ion waves at small $k \lambda_{D}$. Such waves will be very weakly damped, as the ratio of their phase velocity to the ion thermal velocity will be larger than it is for ordinary acoustic waves. The phase velocity of the ion waves then decreases as $\omega$ approaches $\omega_{p i}$, even though $k \lambda_{D}$ is much smaller than unity. However, this group of waves with $\omega \sim \omega_{p i}$ may also be very weakly damped, when $T_{i}$ is indeed negligible so that the ion thermal velocity is quite small.

As we assume that $T_{i}$ is negligible throughout the following applications, it is worthwhile to write down the validity condition for this. It is

$$
\frac{T_{i}}{T_{e}} \ll \frac{1}{3} \frac{\left(k^{2} \lambda_{D}^{2}+k_{z}^{2} \lambda_{S}^{2}\right)}{k^{2} \lambda_{D}^{2}\left(1+k^{2} \lambda_{D}^{2}+k_{z}^{2} \lambda_{S}^{2}\right)} .
$$

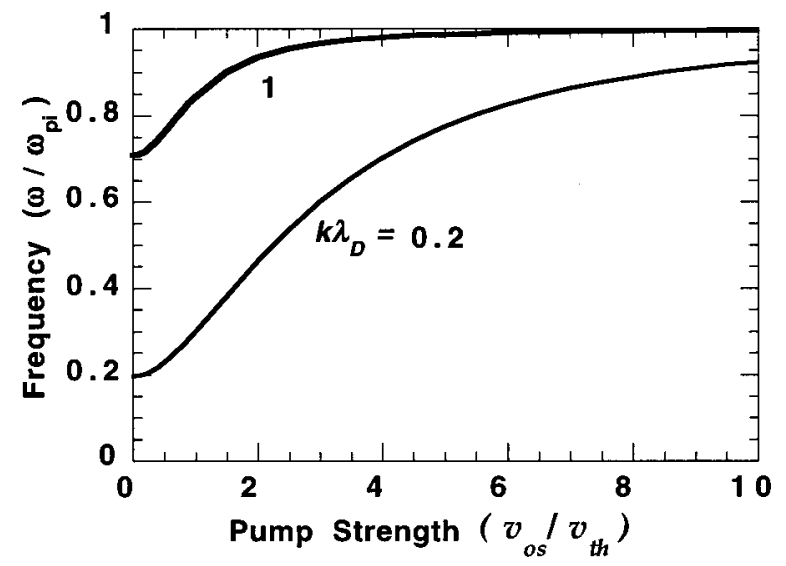

FIG. 2. The relation of frequency and pump strength for the induced ion plasma wave dispersion relation $\left(D_{i}=0\right)$ is shown for the indicated values of $k \lambda_{D}$.

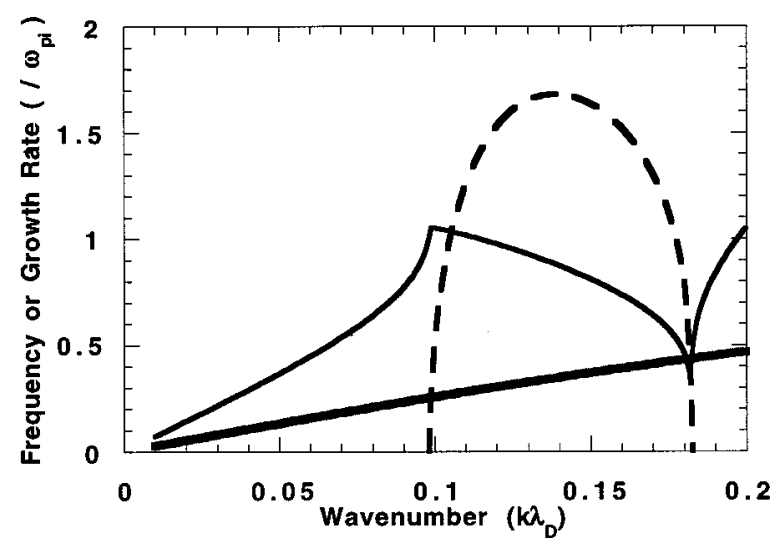

FIG. 3. The ion wave frequency (thin solid line) and growth rate (dashed line) are shown for $k_{0}=0, v_{\text {os }} / v_{\text {th }}=2, n_{c}=1.1 n$, and $\theta=0$. The thick solid line shows the uncoupled ion wave frequency given by $D_{i}=0$. The frequencies and growth rates are normalized to $\omega_{p i}$ here and in Figs. 4 and 5.

The fundamental conclusions reached below are not altered when $T_{i}$ is not negligible, unless $T_{i}$ becomes so large that the ion pressure exceeds the sum of the electron pressure and the energy density of the oscillating electrons.

Figure 2 shows how the wave frequency varies with $v_{\text {os }} / v_{\text {th }}$, for two values of $k \lambda_{D}$. This result should be valid if the density is low enough that the coupling to the Langmuir waves is negligible. For example, and subject to the caveats discussed above, Eqs. (8)-(15) imply that this coupling is negligible at $n=0.1 n_{c}$, for $v_{\text {os }} / v_{\text {th }}=10$, over at least the range $0<k \lambda_{D}<2$. In this case, when $k \lambda_{D}$ is small, electron oscillations can readily produce large increases in the ion wave frequency. When $k \lambda_{D}$ is large, this frequency increase is much reduced.

The response of the fully coupled system, including the Langmuir waves, matters at higher density. We examined this regime as described in the Appendix, using the formalism developed, for example, in Nishikawa ${ }^{24}$ or in Bardwell and Goldman, ${ }^{28}$ to solve the combined dispersion relation for the ion wave and the Stokes and anti-Stokes waves. We did this so as to obtain the growth rate and ion-wave frequency as $k \lambda_{D}$ varies, for chosen values of $v_{\text {os }} / v_{\text {th }}, k_{0} / k, n / n_{c}$, and $\theta$ (the angle between $\mathbf{k}$ and $\mathbf{v}_{\mathrm{os}}$ ). The results are symmetric in

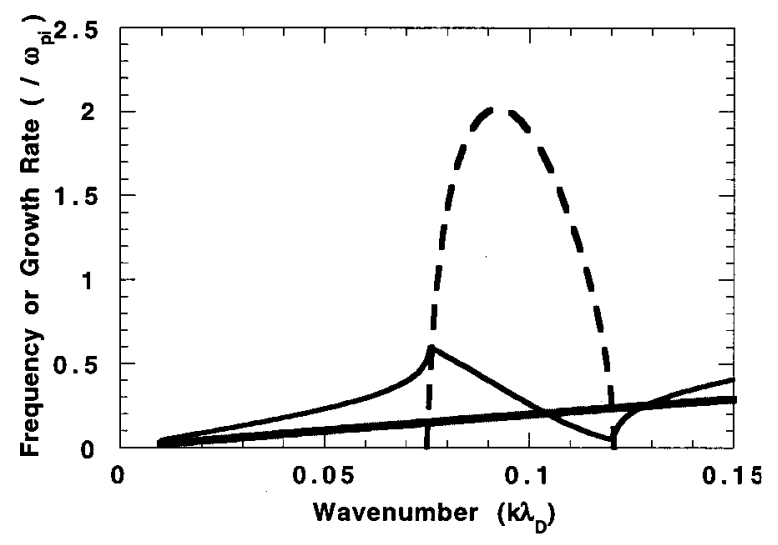

FIG. 4. The ion wave frequency (thin solid line) and growth rate (dashed line) are shown for $k_{0}=k, v_{\text {os }} / v_{\text {th }}=2, n_{c}=1.1 n$, and $\theta=0$. The thick solid line shows the uncoupled ion wave frequency given by $D_{i}=0$. 


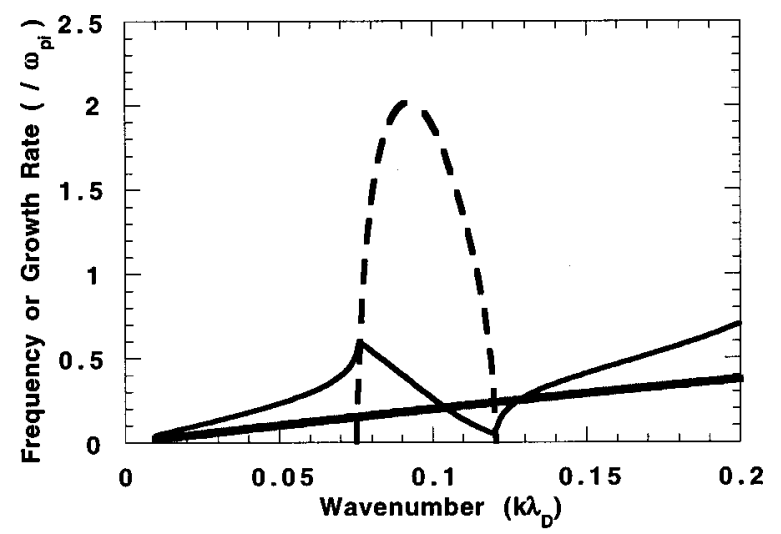

FIG. 5. The ion wave frequency (thin solid line) and growth rate (dashed line) are shown for $k_{0}=k, v_{\text {os }} / v_{\text {th }}=2, n_{c}=1.1 n$, and $|\theta|=45^{\circ}$. The thick solid line shows the uncoupled ion wave frequency given by $D_{i}=0$.

$\theta$ about $\theta=0$. Figures $3-5$ show typical results. Figure 3 shows the result for $k_{0}=0$. One sees that the four-wave coupling does shift the frequency of the ion wave compared to the uncoupled $\left(D_{i}=0\right)$ result, but only by up to about a factor of 2 . There is also a range of $k$ over which the coupled modes are unstable, and would be expected to grow. The growth rate is large, having a maximum in excess of $\omega_{p i}$. For example, near critical density for a laser of $0.5 \mu \mathrm{m}$ wavelength, $\omega_{p i} \sim 10^{14} \mathrm{~s}^{-1}$. This has the consequence that such waves could grow to observable amplitude during a 100 fs laser pulse.

Figures 4 and 5 show results for larger $k$, for angles of $\theta=0^{\circ}$ and $\theta=45^{\circ}$, respectively. Figure 4 shows that a narrower range of $k$ is unstable for $k_{0}=k$, as opposed to for $k_{0}=0$ as shown in Fig. 3. Figure 5 shows that both the values and the range of unstable $k$ are smaller for $k_{0}=k$ and $\theta$ $=45^{\circ}$. The qualitative features are the same in these cases as in the case of Fig. 3. Indeed, the qualitative structure of the solution remains the same as $k_{0}, v_{\text {os }} / v_{\text {th" }}$ and $\theta$ vary. Only the values and range where the waves are unstable change. As $n$ decreases, the range of unstable $k$ also decreases. Eventually, at low enough $n$ for a given value of $v_{\text {os }} / v_{\text {th }}$, no waves are unstable through this process. (Such waves might be driven to large amplitude by other mechanisms, however, such as stimulated scattering.)

We can, in addition, compare the results of this theory with published results of kinetic theory for the experiments ${ }^{19,20}$ of Bernard et al. We will designate the first report ${ }^{19}$ of these experiments as paper B1. In this case, which used a $10 \mu \mathrm{m}$ wavelength laser as a pump and $90^{\circ}$ scattering of a $0.53 \mu \mathrm{m}$ wavelength laser as a probe, the assumption $k_{0} \ll k$ is a good one, so the kinetic theory does apply. Figures 6(a) and 6(b) show the solution of Eqs. (8)-(15) for the two cases represented by Figs. 4(a) and 4(b), respectively, of paper B1. This solution is plotted for three temperatures (150 $\mathrm{eV}, 200 \mathrm{eV}$, and $300 \mathrm{eV}$ ). For comparison, the solution of $D_{i}=0$ is shown for the $200 \mathrm{eV}$ case (as a dashed line). In Fig. 6, $\omega$ is normalized to " $\omega_{i a}$," which designates the frequency of the waves that propagate perpendicular to $\mathbf{v}_{\mathrm{os}}$. This is the normalization used in paper B1. In contrast, in Figs. $1-5 \omega$ is normalized to $\omega_{p i}$. There are several points to be made about these figures.

First, the solution to the fluid equations shown here is qualitatively and quantitatively in good (but not perfect) agreement with the results of kinetic theory presented in paper B1. This is as we would expect, based on the discussion at the end of Sec. IV. The frequencies of the Langmuir waves given by the fluid theory will not be accurate for the conditions of $0.5 \leqslant k \lambda_{D} \leqslant 1$ present here. However, the impact of this error on the ion wave frequency should not be (and is not) large.

Second, the limiting frequency seen in Fig. 6 and in paper $\mathrm{B} 1$ is in all cases the ion plasma frequency. (The ratio of $\omega_{p i}$ to $\omega_{i a}$ varies with $k \lambda_{D}$.) The effect of the pump is to frustrate the Debye shielding until the ion oscillations become essentially unshielded, at which point their frequency approaches $\omega_{p i}$. This interpretation is strengthened by the comparison between the dashed and solid curves in Fig. 6 . The coupling to Langmuir waves does not have significant effects when $v_{\text {os }} / v_{\text {th }}$ is large enough. These physical points, regarding how and why the frequency of the ion waves
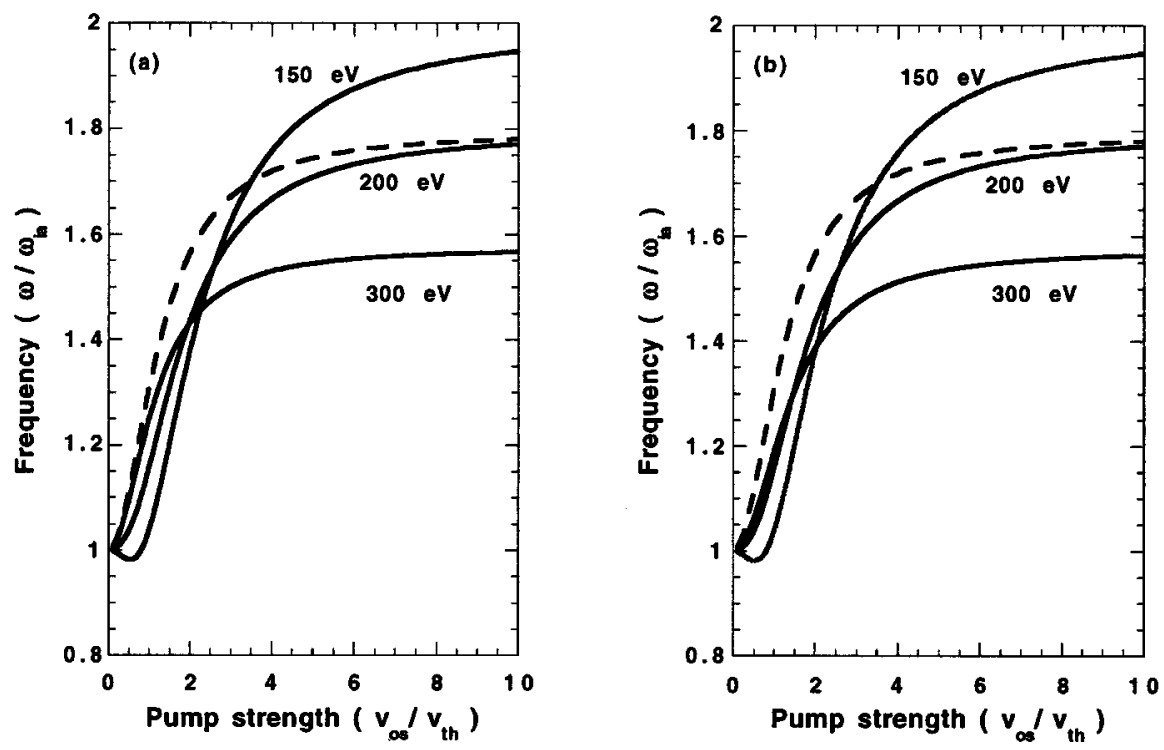

FIG. 6. Frequencies calculated (and normalized) to correspond to Figs. 4(a) and 4(b) in Bernard et al. (Ref. 19). The ion wave frequency is normalized to the frequency of the ion waves with $\mathbf{k} \| \mathbf{k}_{0}$ and is plotted against pump strength $\left(v_{\text {os }} / v_{\text {th }}\right)$ for the electron temperatures indicated. The dashed curves show the frequency determined for $200 \mathrm{eV}$ using $D_{i}=0$, which excludes the effects of coupling to Langmuir waves. (a) $0.6 n_{c}$; (b) $0.7 n_{c}$. 
saturates, were not made in any of the prior work on this subject.

Third, the comparison between the dashed curves and the solid curves in Fig. 6 allows us to understand the shape of the more complete solutions at low $v_{\text {os }} / v_{\text {th }}$, both here and in paper B1. At low $v_{\text {os }} / v_{\text {th }}$, the coupling to the Langmuir waves has the effect of decreasing the ion-wave frequency. This effect is larger for smaller $k \lambda_{D}$. This is not surprising because the four-wave interaction becomes stronger as $k \lambda_{D}$ decreases. $^{32,33}$ In the fluid theory, for the regime shown in Fig. 6, the coupled waves are not predicted to grow unstably. However, at even lower $k \lambda_{D}$, we find that the modulational instability, ${ }^{34}$ corresponding to $\omega=0$, becomes unstable. This instability is also identified and discussed in the second paper $^{20}$ by Bernard et al., where it is referred to as the oscillating two stream instability. This could contribute to the onset of strong Langmuir turbulence ${ }^{34}$ in experiments that last long enough.

\section{CONCLUSION}

Thus, we conclude that any experiment in which driven electron oscillations become significant will observe an increase in the frequency of ion waves having finite $k_{z}$. Even ion waves having very small values of $k \lambda_{D}$, which would ordinarily be thought to be ion acoustic waves, may turn out to have frequencies approaching the ion plasma frequency, as a result of the frustration of Debye shielding. This may explain the satellites that have been observed in harmonic emission, ${ }^{35}$ and could be seen in other data, having frequencies near the ion plasma frequency, and for which no other explanation has been offered. Specifically, the unstable response of the electrons and ions in the presence of the pump will produce ion density modulations with frequencies and growth rates near $\omega_{p i}$. These waves have time to grow during a 100 fs laser pulse, whereas the corresponding acoustic waves would not. They in turn will produce satellites in any wave scattering process.

We note also that any source of large, high-frequency electron oscillations will have the effect discussed here. In this case such oscillations will act as pump waves in the context of the above derivation. Thus, the presence of large amplitude, electron-plasma waves, driven for example by stimulated Raman scattering, will shift the frequency of the ion waves in the region where the Raman occurs. [However, to treat longitudinal pumps quantitatively, which is beyond our scope here, it will be necessary to replace Eq. (10) with the correct expression for $F_{s}$ for this case.] This may explain the observations that stimulated Raman scattering and stimulated Brillouin scattering do not tend to overlap in time and space $^{36}$ and tend not to both be large simultaneously. ${ }^{37}$ To the extent that the plasma waves vary rapidly in time and space, the rapid change in the ion wave frequency provides a mechanism by which Raman can detune and quench Brillouin. (Guzdar and $\mathrm{Sen}^{22}$ proposed that the spatial structure of the pump could quench Brillouin for similar reasons.) In addition, this effect may explain the remarkably large "temperatures" inferred from the frequency of ion waves in some underdense target experiments containing substantial electrostatic turbulence. ${ }^{38}$

In conclusion, oscillating electrons cannot shield iondensity fluctuations over distances smaller than the distance they sample in a plasma period. The consequence is that any such ion-density fluctuations have an increased frequency, of order the ion plasma frequency. The weakly coupled equation for the resulting ion wave frequency $\left[D_{i}=0\right.$ from Eq. (15)] gives an easily evaluated result that should be suitable for a wide range of experimental applications. Such induced ion plasma waves may have contributed to several observations reported in the literature, and may in particular have an impact on ongoing and future work with short pulse lasers.

More generally, in the presence of an intense pump, the phenomenon of frustrated Debye shielding will affect any other mechanism that depends on Debye shielding in the first place. This could potentially include atomic transitions, bremsstrahlung interactions, and synchrotron emission.

\section{ACKNOWLEDGMENTS}

R.P.D. acknowledges support from the U.S. Department of Energy and the University of Michigan. R.S.M. acknowledges support from the Natural Sciences and Engineering Research Council of Canada.

\section{APPENDIX: THE FOUR-WAVE ANALYSIS}

We employed the four-wave theory of DuBois and Goldman, ${ }^{27}$ Nishikawa, ${ }^{24}$ and Bardwell and Goldman ${ }^{28}$ to evaluate the frequency and growth rate of the ion waves. In this analysis, one writes the solution of Eqs. (8)-(15) in the following form:

$$
\begin{aligned}
(2+ & \delta \Omega-i \gamma)(\delta \Omega-i \gamma) \\
& =Q\left\{\frac{1}{(-\delta \Omega+i \gamma+\Delta)}+\frac{1}{(2+\delta \Omega-i \gamma+\Delta+h)}\right\} .
\end{aligned}
$$

Here $\gamma$ is the growth rate of the unstable waves and $\delta \Omega$ represents the deviation of the real ion-wave frequency from $\Omega_{R}$, normalized by $\Omega_{R}$, where $\Omega_{R}$ is the ion-wave frequency in the absence of the four-wave coupling, found by setting $D_{i}=0$. The coefficient $\Delta$, also normalized by $\Omega_{R}$, is the difference between the actual Stokes wave frequency, found by subtracting the ion-wave frequency from the pump frequency, and the resonant stokes wave frequency, found by setting $D_{S}=0$. The sum $(\Delta+h)$, also normalized by $\Omega_{R}$, is the difference between the actual Antistokes wave frequency, found by adding the ion-wave frequency to the pump frequency, and the resonant anti-Stokes wave frequency, found by setting $D_{A}=0$. The coupling coefficient is $Q$.

In terms of the definitions in the main text, several of these quantities can be written as follows:

$$
\begin{aligned}
& k \lambda_{\mathrm{eff}}=k \lambda_{D} \sqrt{1+\frac{k_{z}^{2}}{k^{2}} \frac{v_{\mathrm{os}}^{2}}{v_{\mathrm{th}}^{2}} F_{s}^{2}}, \\
& \Omega_{R}=\omega_{p i} \sqrt{\frac{k^{2} \lambda_{\mathrm{eff}}^{2}}{1+k^{2} \lambda_{\mathrm{eff}}^{2}}+3 k^{2} \lambda_{D}^{2} \frac{T_{i}}{T_{e}}},
\end{aligned}
$$




$$
\begin{aligned}
Q= & \frac{v_{\mathrm{os}}^{2}}{v_{\mathrm{th}}^{2}} \frac{\omega_{\mathrm{pe}}}{\omega_{p i}} \\
& \times \frac{\cos ^{2}(\theta)\left(k \lambda_{D}\right)^{2} \sqrt{n_{c} / n}\left(1+k^{2} / k_{s}^{2}\right)}{8\left(1+k^{2} \lambda_{\text {eff }}^{2}\right)^{2}\left(\frac{k^{2} \lambda_{\text {eff }}^{2}}{1+k^{2} \lambda_{\text {eff }}^{2}}+3 k^{2} \lambda_{D}^{2} \frac{T_{i}}{T_{e}}\right)^{3 / 2} \sqrt{1+3 k_{s}^{2} \lambda_{D}^{2}}},
\end{aligned}
$$

$\Delta=\frac{\omega_{\mathrm{pe}}}{\Omega_{R}}\left(\sqrt{n_{c} / n}-\sqrt{1+3 k_{s}^{2} \lambda_{D}^{2}}\right)-1$,

and

$$
h=\frac{-6\left(\mathbf{k} \cdot \mathbf{k}_{0}\right) v_{\mathrm{th}}^{2}}{\Omega_{R} \omega_{\mathrm{pe}} \sqrt{1+3 k_{s}^{2} \lambda_{D}^{2}}},
$$

with

$$
\operatorname{Re}(\omega)=(1+\delta \Omega) \Omega_{R} .
$$

Given a set of plasma and pump parameters and $\mathbf{k}$, one can calculate $\Omega_{R}, Q, \Delta$, and $h$. One can then solve Eq. (A1) to obtain $\delta \Omega$ and $\gamma$. This is the fundamental calculation that produced Figs. 3-6 in the present paper.

${ }^{1}$ L. Tonks and I. Langmuir, Phys. Rev. 33, 195 (1929).

${ }^{2}$ P. Krstic and Y. Hahn, J. Quant. Spectrosc. Radiat. Transf. 55, 499 (1996).

${ }^{3}$ V. N. Tsytovich, Phys. Usp. 38, 87 (1995).

${ }^{4}$ D. I. Kaltchev and E. A. Perelstein, Phys. Fluids B 5, 164 (1993).

${ }^{5}$ M. S. Dimitrijevic, A. A. Mihajlov, A. Djuric, and B. Grabowski, J. Phys. B 22, 3845 (1989).

${ }^{6}$ B. D. Fried and R. W. Gould, Phys. Fluids 4, 139 (1961).

${ }^{7}$ B. S. Bauer, R. P. Drake, K. G. Estabrook, J. F. Camacho, R. G. Watt, M. D. Wilke, G. Busch, S. Caldwell, and S. A. Baker, Phys. Plasmas 2, 2207 (1995).

${ }^{8}$ B. S. Bauer, R. P. Drake, K. G. Estabrook, R. G. Watt, M. D. Wilke, and S. A. Baker, Phys. Rev. Lett. 74, 3604 (1995).

${ }^{9}$ G. Dimonte, Phys. Rev. Lett. 46, 26 (1981).

${ }^{10}$ Y. M. Aliev and V. P. Silin, Sov. Phys. JETP 21, 601 (1965).

${ }^{11}$ V. P. Silin, Sov. Phys. JETP 21, 1127 (1965).

${ }^{12}$ P. K. Kaw and J. M. Dawson, Phys. Fluids 13, 2586 (1969).

${ }^{13}$ V. P. Silin, Sov. Phys. JETP 24, 1242 (1967).

${ }^{14}$ L. M. Gorbunov, Sov. Phys. JETP 28, 1220 (1969).

${ }^{15}$ J. R. SanMartin, Phys. Fluids 13, 1533 (1970).
${ }^{16}$ W. Rozmus, Y. Al-Shiraida, and A. A. Offenberger, Phys. Fluids 27, 589 (1984).

${ }^{17}$ W. Rozmus, W. Tighe, A. A. Offenberger, and K. Estabrook, Phys. Fluids 28, 920 (1985).

${ }^{18}$ P. Mounaix, These de Docteur en Sciences, Universite de Paris-Sud, Centre d'Orsay, 1990.

${ }^{19}$ J. E. Bernard, H. A. Baldis, D. M. Villeneuve, A. B. Langdon, and W. Rozmus, Phys. Rev. Lett. 58, 1644 (1987).

${ }^{20}$ J. E. Bernard, H. A. Baldis, D. M. Villeneuve, A. B. Langdon, and W. Rozmus, Phys. Rev. A 39, 2549 (1989).

${ }^{21}$ A. A. Vedenov, A. V. Gordeev, and L. I. Rudakov, Plasma Phys. 9, 719 (1967).

${ }^{22}$ P. N. Guzdar and A. Sen, Nucl. Fusion 18, 970 (1978).

${ }^{23}$ Note that $T_{e}$ as defined here may be altered, although not very much, as kinetic strong-pump effects change the symmetric shape of the electron distribution: A. B. Langdon, Phys. Rev. Lett. 44, 575 (1980); B. B. Afeyan, A. E. Chou, J. P. Matte et al., ibid. 80, 2322 (1998).

${ }^{24}$ K. Nishikawa, J. Phys. Soc. Jpn. 24, 916 (1968).

${ }^{25} \mathrm{~W}$. L. Kruer, The Physics of Laser Plasma Interactions (Addison-Wesley, Redwood City, 1988).

${ }^{26}$ D. F. DuBois and M. V. Goldman, Phys. Rev. 164, 207 (1967).

${ }^{27}$ D. F. DuBois and M. V. Goldman, Phys. Rev. Lett. 19, 1105 (1967).

${ }^{28}$ S. Bardwell and M. V. Goldman, Astrophys. J. 209, 912 (1976).

${ }^{29}$ M. V. Goldman, D. L. Newman, D. Russell, D. F. DuBois, H. Rose, R. P. Drake, and A. M. Rubenchik, Phys. Plasmas 2, 1947 (1995).

${ }^{30}$ R. P. Drake, M. V. Goldman, and J. S. D. Groot, Phys. Plasmas 1, 2448 (1994).

${ }^{31}$ K. Nishikawa and C. S. Liu, in Advances in Plasma Physics, edited by A. Simon and W. Thompson (Interscience, New York, 1976), Vol. 6, p. 1.

${ }^{32}$ D. F. DuBois, H. A. Rose, and D. Russell, J. Geophys. Res. 95, 21221 (1990).

${ }^{33}$ D. F. DuBois, H. A. Rose, and D. Russell, Phys. Rev. Lett. 66, 1970 (1991).

${ }^{34}$ M. V. Goldman, Rev. Mod. Phys. 56, 709 (1984).

${ }^{35}$ L. Zhao, Ph.D. thesis, University of Toronto, 1998; R. S. Marjoribanks, L. Zhao, F. W. Budnik et al., in Proceedings of the International Conference on Superstrong Fields in Plasmas, Varenna, edited by M. Lontano, G. Mourou, F. Pegoraro, and E. Sindoni (American Institute of Physics, Melville, NY, 1998), Vol. CP426, p. 342.

${ }^{36}$ C. Labaune, H. A. Baldis, E. Schifano, B. S. Bauer, A. Michard, N. Renard, W. Seka, J. D. Moody, and K. Estabrook, Phys. Rev. Lett. 76, 3727 (1996).

${ }^{37}$ D. S. Montgomery, B. B. Afeyan, J. A. Cobble, J. C. Fernandez, M. D. Wilke, S. H. Glenzer, R. K. Kirkwood, B. J. MacGowan, J. D. Moody, E. L. Lindman, D. H. Munro, B. H. Wilde, H. A. Rose, D. F. Dubois, B. Bezzerides, and H. X. Vu, Phys. Plasmas 5, 1973 (1998).

${ }^{38}$ S.-Y. Chen, M. Krishnan, A. Maksimchuk, and D. Umstadter, Phys. Plasmas 7, 403 (2000). 